\title{
Accelerating Kernel Classifiers Through Borders Mapping
}

\author{
Peter Mills \\ peteymills@hotmail.com
}

January 31, 2023

\begin{abstract}
Support vector machines (SVM) and other kernel techniques represent a family of powerful statistical classification methods with high accuracy and broad applicability. Because they use all or a significant portion of the training data, however, they can be slow, especially for large problems. Piecewise linear classifiers are similarly versatile, yet have the additional advantages of simplicity, ease of interpretation and, if the number of component linear classifiers is not too large, speed. Here we show how a simple, piecewise linear classifier can be trained from a kernel-based classifier in order to improve the classification speed. The method works by finding the root of the difference in conditional probabilities between pairs of opposite classes to build up a representation of the decision boundary. When tested on 17 different datasets, it succeeded in improving the classification speed of a SVM for 12 of them by up to two orders-of-magnitude. Of these, two were less accurate than a simple, linear classifier. The method is best suited to problems with continuum features data and smooth probability functions. Because the component linear classifiers are built up individually from an existing classifier, rather than through a simultaneous optimization procedure, the classifier is also fast to train.
\end{abstract}

\section{Keywords}

class borders, multi-dimensional root-finding, binary classifier, multiclass classifier, kernel methods, non-parametric statistics 


\section{Contents}

1 Introduction

2 Theory 4

2.1 Support vector machines . . . . . . . . . . . . 5

2.2 Borders classification . . . . . . . . . . . . . 6

2.3 Multi-class classification . . . . . . . . . . . . . 8

2.4 Skill scores . . . . . . . . . . . . . . . 9

3 Software and data $\quad \mathbf{1 0}$

3.1 LIBLINEAR . . . . . . . . . . . . . . . . . . 10

3.2 LIBSVM . . . . . . . . . . . . . . . . . . . . . . . . . . . . . . . . . . . . .

3.3 LibAGF ...................... . . 11

3.4 Datasets . . . . . . . . . . . . . . . . 11

4 A simple example 13

5 Case studies $\quad 22$

6 Conclusions

A Sub-sampling 31

References 33

\section{Introduction}

Linear classifiers are well studied in the literature. Methods such as the perceptron, Fisher discriminant, logistic regression and now linear support vector machines (SVMs) (Michie et al., 1994) are often appropriate for relatively simple, binary classification problems in which both classes are closely clustered or otherwise well separated. An obvious extension for more complex problems is a piecewise linear classifier in which the decision boundary is built up from a series of linear classifiers. Piecewise linear classifiers enjoyed some popularity during the early development of the field of machine learning (Osborne, 1977; Sklansky and Michelotti, 1980; Lee and Richards, 1984, 1985) and because of their versatility, generality and simplicity there has been recent renewed interest (Bagirov, 2005; Kostin, 2006; Gai and Zhang, 2010; Webb, 2012; Wang and Saligrama, 2013; (Pavlidis et al., 2016). 
A linear classifier takes the form:

$$
g(\vec{x})=\vec{v} \cdot \vec{x}+b
$$

where $\vec{x}$ is a test point in the feature space, $\vec{v}$ is a normal to the decision hyper-surface, $b$ determines the location of the decision boundary along the normal and $g$ is the decision function which we use to estimate the class of the test point through its sign.

A piecewise linear classifier collects a set of such linear classifiers: $\left\{\vec{v}_{i}\right\}=$ $\left\{\vec{v}_{1}, \vec{v}_{2}, \vec{v}_{3} \ldots\right\} ;\left\{b_{i}\right\}=\left\{b_{1}, b_{2}, b_{3}, \ldots\right\}$. The two challenges here are, first, how to efficiently train each of the decision boundaries and, second, the related problem of how to partition the feature space to determine which linear decision boundary is used for a given test point.

In Bagirov (2005) for instance, the decision function is defined by dividing the set of linear classifiers and maximizing the minimum linear decision value in each subset. To train the classifier, a cost function is defined in terms of this decision function and directly minimized using an analog to the derivative for non-smooth functions (Bagirov, 1999). Naturally, such an approach will be quite computationally costly, especially for a large number of component linear classifiers.

Partitioning of the feature space can be separate from the discrimination borders (Huang et al., 2013) but more normally the discrimination borders are themselves sufficient to partition the feature space (Osborne, 1977; Lee and Richards, 1984; Bagirov, 2005; Kostin, 2006). This means that all or a significant fraction of the component linear classifiers must be evaluated. In Kostin (2006), for instance, the linear classifiers form a decision tree.

In the method described in this paper, the constant term, $b_{i}$, is changed to a vector and the partitioning accomplished through a nearest neighbours to this vector. Thus the zone of influence for each hyperplane will be described by the Veronoi tessellation (Kohonen, 2000). If the class domains are simply connected and don't curve back on themselves, then the partitions will also be shaped as hyper-pyramids, with the axes of the pyramids roughly perpendicular to the decision border. A dot product with each of the vectors must be calculated, similar to a linear classifier, but afterwards only a single linear decision function is evaluated.

There seems to be some tension in the literature between training the decision boundary through simultaneous optimization (Bagirov, 2005; Wang and Saligrama, 2013 ) or through methods that are more piece meal (Gai and Zhang, 2010; Herman and Yeung, 1992; Kostin, 2006). Obviously, simultaneous optimiza- 
tion will be more accurate but also more computationally expensive. In addition, finding global minima for cost functions involving more than a handful of hyper-surfaces will be all but impossible. There is also the issue of separability. Many of the current methods seem designed with disjoint classes in mind (Herman and Yeung, 1992), for instance Gai and Zhang (2010), who stick the hyper-plane borders between neighbouring pairs of opposite classes. Yet there is no reason why a piecewise linear classifier cannot be just as effective for overlapping classes.

The technique under discussion in this paper mitigates all of these issues because it is not a stand-alone method but requires estimates of the conditional probabilities. It is used to improve the time performance of kernel methods, or for that matter, any binary classifier that returns a continuous decision function that can approximate a conditional probability. This is done while maintaining, in all but a few cases, most of the accuracy.

Several of the piecewise linear techniques found in the literature work by positioning each hyperplane between pairs of clusters or pairs of training samples of opposite class (Sklanskv and Michelotti, 1980; Tenmoto et al., 1998; Kostin, 2006; Gai and Zhang, 2010). Other unsupervised or semisupervised classifiers work by placing the hyperplanes in regions of minimum density (Pavlidis et al., 2016). The method described in this paper in some senses combines these two techniques by finding the root of the difference in conditional probabilities along a line between two points of opposite class. It will be tested on a kernel-based classifier called a support vector machine (SVM) (Michie et al., 1994; Müller et al., 2001) and evaluated based on how well it improves classification speed and at what cost to accuracy. It will also be compared to a simple, linear classifier as in (11).

Section 2 describes the theory of support vector machines and linear classifiers as well as the piecewise linear classifier or "borders" classifier that will be trained on the SVM. Section 3 describes the software and test datasets then in section 4 we analyze the different classification algorithms on a simple, synthetic dataset. Section 5 outlines the results for 17 case studies while in Section 5 we discuss the results. Section 6 concludes the paper.

\section{Theory}

We are interested in training a binary classification model based on a set of training data, $\left\{\vec{x}_{i}: y_{i} \mid i \in[1, n]\right\}$, where $\vec{x}_{i}$ is a vector of features data and $y_{i} \in\{-1,+1\}$ is the corresponding class label. In this section we will 
describe methods for training the three types of models tested in this paper: a basic linear classifier, a support vector machine (SVM) and a borders classifier.

\subsection{Support vector machines}

Even though different software is used for each, we describe support vector machines (SVMs) and linear classifiers in the same section since a linear classifier is just a restricted version of a SVM. The decision function for a SVM is defined as follows:

$$
g_{\text {svm }}(\vec{x})=\sum_{i=1}^{n} w_{i} y_{i} K\left(\vec{x}, \vec{x}_{i}\right)+b
$$

where $K$ is a kernel function, $b$ is a constant, $\left\{w_{i}\right\}$ are a sparse set of coefficients and $n$ is the number of training samples. The class is determined as in (11) by the sign of the decision value:

$$
c(\vec{x})=\frac{g_{\text {svm }}(\vec{x})}{\left|g_{\text {svm }}(\vec{x})\right|}
$$

where for convenience, the class labels are defined as $c \in\{-1,1\}$.

The constant and coefficients are fitted by solving the following, quadratic optimization problem:

$$
\max _{\left\{w_{i}\right\}}\left[\sum_{i} w_{i}-\frac{1}{2} \sum_{i, j} w_{i} w_{j} y_{i} y_{j} K\left(\vec{x}_{i}, \vec{x}_{j}\right)\right]
$$

subject to the following constraints:

$$
\begin{aligned}
0 \leq w_{i} & \leq C \\
\sum_{i} w_{i} y_{i} & =0
\end{aligned}
$$

where $C$ is the cost (Müller et al., 2001; Chang and Lin, 2011). The coefficients thus derived are typically sparse and the training samples for which $w_{i}$ are non-zero are called support vectors.

SVM models will be trained with LIBSVM (Chang and Lin, 2011). LIBSVM has the option to return estimates of the conditional probabilities by transforming the raw decision function using logistic regression:

$$
\tilde{r}_{\text {svm }}(\vec{x})=\tanh \left(\frac{A g_{\text {svm }}(\vec{x})+B}{2}\right)
$$


where $A$ and $B$ are coefficients derived from the training data via a nonlinear fitting technique (Platt, 1999; Lin et al., 2007) and $\tilde{r}_{\text {svm }}$ is an estimator for the difference in conditional probabilities-see Equation (10), below. Probability estimates are needed for the borders training: see the following section.

In a linear SVM, the kernel function, $K$, is a simple dot product:

$$
g_{s v m}(\vec{x})=\sum_{i} w_{i} y_{i} \vec{x} \cdot \vec{x}_{i}+b
$$

By exchanging the order of the summation operators, we can show that the normal to the decision hyper-plane, $\vec{v}$, takes on the following value:

$$
\vec{v}=\sum_{i} w_{i} y_{i} \vec{x}_{i}
$$

with which classifications may be performed using the simpler formula in (11).

\subsection{Borders classification}

In kernel SVM, the decision border exists only implicitly in a hypothetical, abstract space. Even in linear SVM, if the software is generalized to recognize the simple dot product as only one among many possible kernels, then the decision function may be built up, as in (2) through a sum of weighted kernels. This is the case for LIBSVM. The advantage of an explicit decision border as in (11) is that it is fast. The problem with a linear border is that, except for a small class of problems, it is not very accurate.

In the binary classification method described in Mills (2011), a non-linear decision border is built up piece-wise from a collection of linear borders. It is essentially a root-finding procedure for a decision function, such as $\tilde{r}_{\text {svm }}$ in (17). Let $\tilde{r}$ be a decision function that approximates the difference in conditional probabilities:

$$
\tilde{r}(\vec{x}) \approx r(\vec{x})=p(+1 \mid \vec{x})-p(-1 \mid \vec{x})
$$

where $p(c \mid \vec{x})$ represents the conditional probabilities of a binary classifier having labels $c \in\{-1,+1\}$.

The procedure is as follows: pick a pair of points on either side of the decision boundary (the decision function has opposite signs). Good candidates are one random training sample from each class. Then, zero the decision function along the line between the points. This can be done as many times 
as needed to build up a good representation of the decision boundary. We now have a set of points, $\left\{\vec{b}_{i}\right\}$, such that $\tilde{r}\left(\vec{b}_{i}\right)=0$ for every $i \in\left[1, n_{b}\right]$ where $n_{b}$ is the number of border samples.

Along with the border samples, $\left\{\vec{b}_{i}\right\}$, we also collect a series of normal vectors, $\left\{\vec{v}_{i}\right\}$ such that:

$$
\vec{v}_{i}=\left.\nabla_{\vec{x}} \tilde{r}\right|_{\vec{x}=\vec{b}_{i}}
$$

With this system, determining the class is a two step process. First, the nearest border sample to the test point is found. Second, we define a new decision function, $g_{\text {border }}$, equivalent to (10), through a dot product with the normal:

$$
\begin{aligned}
i & =\arg \min _{j}\left|\vec{x}-\vec{b}_{j}\right| \\
g_{\text {border }}(\vec{x}) & =\vec{v}_{i} \cdot\left(\vec{x}-\vec{b}_{i}\right)
\end{aligned}
$$

The class is determined by the sign of the decision function as in (3). The time complexity is independent of the number of training samples, rather it is linearly proportional to the number of border vectors, $n_{b}$, a tunable parameter. The number required for accurate classifications is dependent on the complexity of the decision border.

The gradient of the SVM decision in (7) is:

$$
\nabla_{\vec{x}} \tilde{r}_{s v m}=\left[1-\tilde{r}_{s v m}^{2}(\vec{x})\right] \sum_{i} w_{i} y_{i} \nabla_{\vec{x}} K\left(\vec{x}, \vec{x}_{i}\right)
$$

Gradients of the initial decision function are useful not only to derive normals to the decision boundary, but also as an aid to root finding when searching for border samples. If the decision function used to compute the border samples represents an estimator for the difference in conditional probabilities, then the raw decision value, $g_{b o r d e r}$, derived from the border sampling technique in (12) can also return estimates of the conditional probabilities with little extra effort and little loss of accuracy, also using a sigmoid function:

$$
\tilde{r}_{\text {border }}(\vec{x})=\tanh \left[g_{\text {border }}(\vec{x})\right]
$$

This assumes that the class posterior probabilities, $p(\vec{x} \mid c)$, are approximately Gaussian near the border (Mills, 2011).

The border classification algorithm returns an estimator, $\tilde{r}_{b o r d e r}$, for the difference in conditional probabilities of a binary classifier using equations (12) and (14). It can be trained with the function in $\tilde{r}_{s v m}$ in (7), or any other continuous, differentiable, non-parametric estimator for the difference 
in conditional probabilities, $r$. At the cost of a small reduction in accuracy, it has the potential to drastically reduce classification time for kernel estimators and other non-parametric statistical classifiers, especially for large training datasets, since it has $O\left(n_{b}\right)$ time complexity instead of $O(n)$ complexity, where $n_{b}$, the number of border samples, is a free parameter. The actual number chosen can trade off between speed and accuracy with rapidly diminishing returns beyond a certain point. One hundred border samples $\left(n_{b}=100\right)$ is usually sufficient. The computation of $\tilde{r}_{b o r d e r}$ also involves very simple operations - floating point addition, multiplication and numerical comparison, with no transcendental functions except for the very last step (which can be omitted) - so the coefficient for the time complexity will be small.

A border classifier trained with SVM will be referred to as an "SVMborders" classifier or an "accelerated" SVM classifier. For more details on the algorithm, please refer to Mills (2011), in particular Sections 2.2, 2.3 and 3.3.

\subsection{Multi-class classification}

The border classification algorithm, like SVM, only works for binary classification problems. It is quite easy to generalize a binary classifier to perform multi-class classifications by using several of them and the number of ways of doing so grows exponentially with the number of classes. Since LIBSVM uses the "one-versus-one" method (Hsu and Lin, 2002) of multi-class classification, this is the one we will adopt.

A major advantage of the borders classifier is that it returns probability estimates. These estimates have many uses including measuring the confidence of as well as recalibrating the class estimates (Mills, 2009, 2011). Thus the multi-class method should also solve for the conditional probabilities in addition to returning the class label.

In a one-vs.-one scheme, the multi-class conditional probabilities can be related to those of the binary classifiers as follows:

$$
r_{i j}(\vec{x})=\frac{p(j \mid \vec{x})-p(i \mid \vec{x})}{p(i \mid \vec{x})+p(j \mid \vec{x})}
$$

where $i \in\left[1, n_{c}-1\right], j \in\left[1, n_{c}\right], j>i, n_{c}$ is the number of classes, and $r_{i j}$ is the difference in conditional probabilities of the binary classifier that discriminates between the $i$ th and $j$ th classes. Wu et al. (2004) transform 
this problem into the following linear system:

$$
\begin{aligned}
p_{i} \sum_{k \mid k \neq i}\left(r_{k i}+1\right)^{2}+\sum_{j \mid j \neq i} p_{j}\left(1-r_{i j}^{2}\right)+\lambda & =0 \\
\sum_{j} p_{j} & =1
\end{aligned}
$$

where $p_{i}=p(i \mid \vec{x})$ is the $i$ th multi-class conditional probability and $\lambda$ is a Lagrange multiplier. They also show that the constraints not included in the problem, that the probabilities are all positive, are always satisfied and describe an algorithm for solving it iteratively, although a simple matrix solver is sufficient.

\subsection{Skill scores}

It is important to evaluate a result based on skill scores that reliably reflect how well a given classifier is doing. Thus we will define the two scores used in this validation exercise since one in particular is not commonly seen in the literature even though it has several attractive features.

Let $\left\{\eta_{i j}\right\}$ be the confusion matrix, that is the number test values for which the first classifier (the "truth") returns the $i$ th class while the second classifier (the estimate) returns the $j$ th class. Let $n_{\text {test }}=\sum_{i} \sum_{j} \eta_{i j}$ be the total number of test points.

The accuracy is given:

$$
a=\frac{\sum_{i} \eta_{i i}}{n_{\text {test }}}
$$

or simply the fraction of correct guesses.

The uncertainty coefficient is a more sophisticated measure based on the channel capacity (Shannon and Weaver, 1963). It has the advantage over simple accuracy in that it is not affected by the relative size of each class distribution. It is also not affected by consistent rearrangement of the class labels.

The entropy of the prior distribution is given:

$$
\begin{aligned}
H_{i} & =-\sum_{i}\left(\sum_{j} \frac{\eta_{i j}}{n_{\text {test }}}\right) \log \left(\sum_{j} \frac{\eta_{i j}}{n_{\text {test }}}\right) \\
& =-\frac{1}{n_{\text {test }}}\left[\sum_{i}\left(\sum_{j} \eta_{i j}\right) \log \left(\sum_{j} \eta_{i j}\right)-\log n_{\text {test }}\right]
\end{aligned}
$$


while the entropy of the posterior distribution is given:

$$
\begin{aligned}
H(i \mid j) & =-\sum_{i} \sum_{j}\left(\frac{\eta_{i j}}{n_{\text {test }}}\right) \log \left(\frac{\eta_{i j}}{\sum_{i} \eta_{i j}}\right) \\
& =-\frac{1}{n_{\text {test }}}\left[\sum_{i} \sum_{j} \eta_{i j} \log \eta_{i j}-\sum_{j}\left(\sum_{i} \eta_{i j}\right) \log \left(\sum_{i} \eta_{i j}\right)\right]
\end{aligned}
$$

The uncertainty coefficient is defined in terms of the prior entropy, $H_{i}$, and the posterior entropy, $H(i \mid j)$, as follows:

$$
U(i \mid j)=\frac{H_{i}-H(i \mid j)}{H_{i}}
$$

and tells us: for a given test classification, how many bits of information on average does the estimate supply of the true class value? (Press et al., 1992; Mills, 2011).

\section{Software and data}

\subsection{LIBLINEAR}

LIBLINEAR is a software package for linear statistical classification developed by the Machine Learning Group at the National Taiwan University. It supports several different training methods including primal and dual SVM as well as logistic regression. The method that provided the most accurate results for each dataset was used. The library may be downloaded at: https://www.csie.ntu.edu.tw/ cjlin/liblinear (Fan et al., 2008). LIBLINEAR is very fast for both training and prediction and it is especially well suited to large-dimensional problems.

\section{$3.2 \quad$ LIBSVM}

LIBSVM is a machine learning software library for support vector machines developed by Chih-Chung Chang and Chih-Jen Lin of the National Taiwan University, Taipei, Taiwan (Chang and Lin, 2011). It includes statistical classification using two regularization methods for minimizing over-fitting: $C-S V M$ and $\nu$-SVM. It also includes code for nonlinear regression and density estimation or "one-class SVM". SVM models were trained using the svm-train command while classifications done with svm-predict. LIBSVM can be found at: https://www.csie.ntu.edu.tw/ cjlin/libsvm. 
Table 1: Summary of datasets used in the numerical trials.

\begin{tabular}{|c|c|c|c|c|c|c|}
\hline & $D$ & Type & $n_{c}$ & Train & Test & Reference \\
\hline heart & 13 & float & 2 & 270 & - & (Lichman, 2013) \\
\hline shuttle & 9 & float & 7 & 43500 & 14500 & King et al., 1995) \\
\hline sat & 36 & float & 6 & 4435 & 2000 & King et al., 1995) \\
\hline segment & 19 & float & 7 & 2310 & - & King et al., 1995) \\
\hline dna & 180 & binary & 3 & 2000 & 1186 & (Michie et al., 1994) \\
\hline splice & 60 & cat & 3 & 1000 & 2175 & (Michie et al., 1994) \\
\hline codrna & 8 & mixed & 2 & 59535 & 271617 & (Uzilov et al., 2006) \\
\hline letter & 16 & integer & 26 & 20000 & - & (Frey and Slate, 1991) \\
\hline pendigits & 16 & integer & 10 & 7494 & 3498 & (Alimoglu, 1996) \\
\hline usps & 256 & float & 10 & 7291 & 2001 & (Hull, 1994) \\
\hline mnist & 665 & integer & 10 & 45000 & 10000 & LeCun et al., 1998) \\
\hline ijcnn1 & 22 & float & 2 & 49990 & 91701 & (Feldkamp and Puskorius, 1998 ) \\
\hline madelon & 500 & integer & 2 & 2000 & 600 & Guvon et al., 2004) \\
\hline seismic & 50 & float & 2 & 78823 & 19705 & Duarte and Hu, 2004) \\
\hline mushrooms & 112 & binary & 2 & 8124 & - & (Iba et al., 1988) \\
\hline phishing & 68 & binary & 2 & 11055 & - & (Mohommad et al., 2014) \\
\hline humidity & 7 & float & 8 & 86400 & - & (Mills, 2009) \\
\hline
\end{tabular}

LIBSVM is not very fast but tends to be very accurate for a wide variety of problems.

\subsection{LibAGF}

Similar to LIBSVM, libAGF is a machine learning library but for variable kernel estimation (Mills, 2011; Terrell and Scott, 1992) rather than SVM. Like LIBSVM, it supports statistical classification, nonlinear regression and density estimation. It supports both Gaussian kernels and knearest neighbours. It was written by Peter Mills and can be found at https://github.com/peteysoft/libmsci. To convert a LIBSVM model to a borders model, the single command, svm_accelerate, can be used. Classifications are then performed with classify_m.

\subsection{Datasets}

The borders classification algorithm was tested on a total of 17 different datasets. These will be briefly described in this section. The collection covers a fairly broad range of size and types of problems, number of classes and 
number and types of attributes but with the focus on larger datasets where the borders technique is actually useful. Four of the datasets are from the "Statlog" project (Michie et al., 1994; King et al., 1995) and are nicknamed "heart", "shuttle", "sat" and "segment". The heart disease ("heart") dataset contains thirteen attributes of 270 patients along with one of two class labels denoting either the presence or absence of heart disease. The dataset comes originally from the Cleveland Clinic Foundation and two versions are stored on the machine learning database of U. C. Irvine (Lichman, 2013).

The shuttle dataset is interesting because the classes have a very uneven distribution meaning that multi-class classifiers with a symmetric breakdown of the classes, such as one-vs.-one, tend to perform poorly. The shuttle dataset comes originally from NASA and was taken from an actual space shuttle flight. The classes describe actions to be taken at different flight configurations.

The satellite ("sat") dataset is a satellite remote-sensing land classification problem. The attributes represent 3-by-3 segments of pixels in a Landsat image with the class corresponding to the type of land cover in the central pixel. The segmentation ("segment") dataset is also an image classification dataset consisting of 3-by-3 pixel sets from outdoor images.

The DNA dataset is concerned with classifying a 60 base-pair sequence of DNA into one of three values: an intron-extron boundary, an extron-intron boundary or neither of those two. That is, during protein creation, part of the sequence is spliced out, with the section kept being the intron and that spliced out being the extron. There are two versions of it: one called "splice" with the original sequence of 4 nucleotide bases but only two classes and one called "dna" in which the features data has been reprocessed so that the 60 base values are transformed to 180 binary attributes but keeping the original three classes (Michie et al., 1994). Another dataset from the field of microbiology is the "codrna" dataset which deals with detection of noncoding RNA sequences (Uzilov et al., 2006).

There are four text-classification datasets: "letter", "pendigits", "usps" and "mnist". The "letter" dataset is a text-recognition problem concerned with classifying a character into one of the 26 letters of the alphabet based on processed attributes of the isolated character (Frey and Slate, 1991). The pendigits dataset is similar to the letter dataset except for classifying numbers instead of letters (Alimoglu, 1996). The "usps" dataset deals with classifying text for the purpose of mailing letters (Hull, 1994). The "mnist" dataset uses 28 by 28 pixel images to classify text into one of ten different characters (LeCun et al., 1998). Pixels that always take on the same value were removed. 
Two of the datasets are machine-learning competition challenges. The "ijcnn1" dataset is from the International Joint Conference on Neural Networks Neural Networks Competition(Feldkamp and Puskorius, 1998) while the "madelon" dataset comes from the International Conference on Neural Information Processing Systems Feature Selection Challenge (Guyon et al., 2004).

The "seismic" dataset deals with vehicle classification from seismic data (Duarte and Hu, 2004). The "mushrooms" dataset classifies wild mushrooms into poisonous and non-poisonous varieties based on their physical characteristics (Iba et al., 1988). The "phishing" dataset uses characteristics of a web address to predict whether or not a website is being used for nefarious purposes (Mohommad et al., 2014).

The final dataset, the "humidity" dataset, comprises simulated satellite radiometer radiances across 7 different frequencies in the microwave range. Corresponding to each instance is a value for relative humidity at a single vertical level. These humidity values have been discretized into 8 ranges to convert it into a statistical classification problem. A full description of the genesis of this dataset as well as a rationale for treatment using statistical classification is contained in Mills (2009). The statistical classification methods discussed in this paper were originally devised specifically for this problem.

Most of the datasets have been supplied already divided into a "test" set and a "training" set. If this is the case, then it is noted in the summary in Table 1 and the data has been used as given with the training set used for training and the test set used for testing. If the data is provided all in one lump, then it was randomly divided into test and training sets with the division different for each of the ten numerical trials.

To provide the best idea of when the technique is effective and when it is not, results from all 17 datasets will be shown. All datasets were preprocessed in the same way: by taking the averages and standard deviations of each feature from the training data and subtracting the averages from both the test and training data and dividing by the standard deviations. Features that took on all the same value in the training data were removed.

\section{A simple example}

We use the pair of synthetic test classes defined in Mills (2011) to illustrate the difference between support vectors and border vectors. Figure 1 shows a realization of the two sample classes in red and blue, comprising 300 samples 


\section{Support vectors}

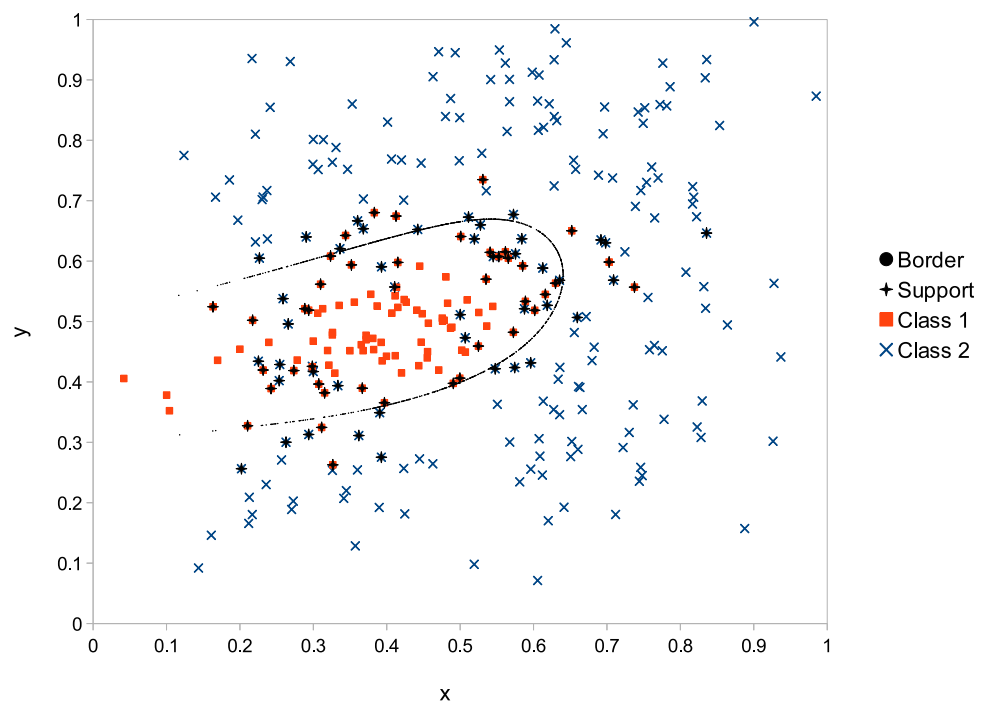

Figure 1: Support vectors for a pair of synthetic test classes. 


\section{Border vectors}

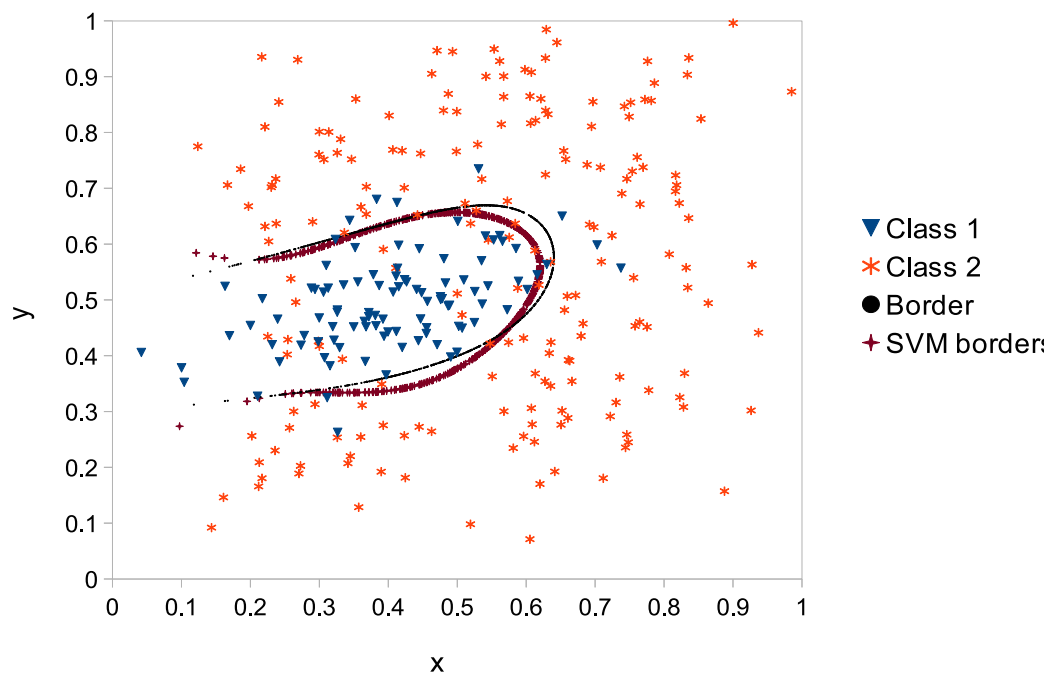

Figure 2: Borders mapped by the border-classification method starting with probabilities from the class definitions and a support vector machine (SVM).

total, along with the support vectors derived from a LIBSVM model. The support vectors are a subset of the training samples and while they tend to cluster around the border, they do not define it. For reference, the border between the two classes is also shown. This has been derived from the border-classification method described in Section 2.2 using the mathematical definition of the classes, hence it represents the "true" border to within a very small numerical error.

The true border is also compared with those derived from LIBSVM probability estimates in Figure 2. The classes are again shown for reference. While these borders contain several hundred samples for a clear view of where they are located using each method, in fact the method works well with surprisingly few samples. Figure 3 shows a plot of the skill versus the number of border samples, where U.C. stands for uncertainty coefficient. Note that the scores saturate at only about 20 samples meaning that for this problem at least, very fast classifications are possible.

Unlike support vectors, the number of border samples required is approximately independent of the number of training samples. In addition to 
Skill versus border samples

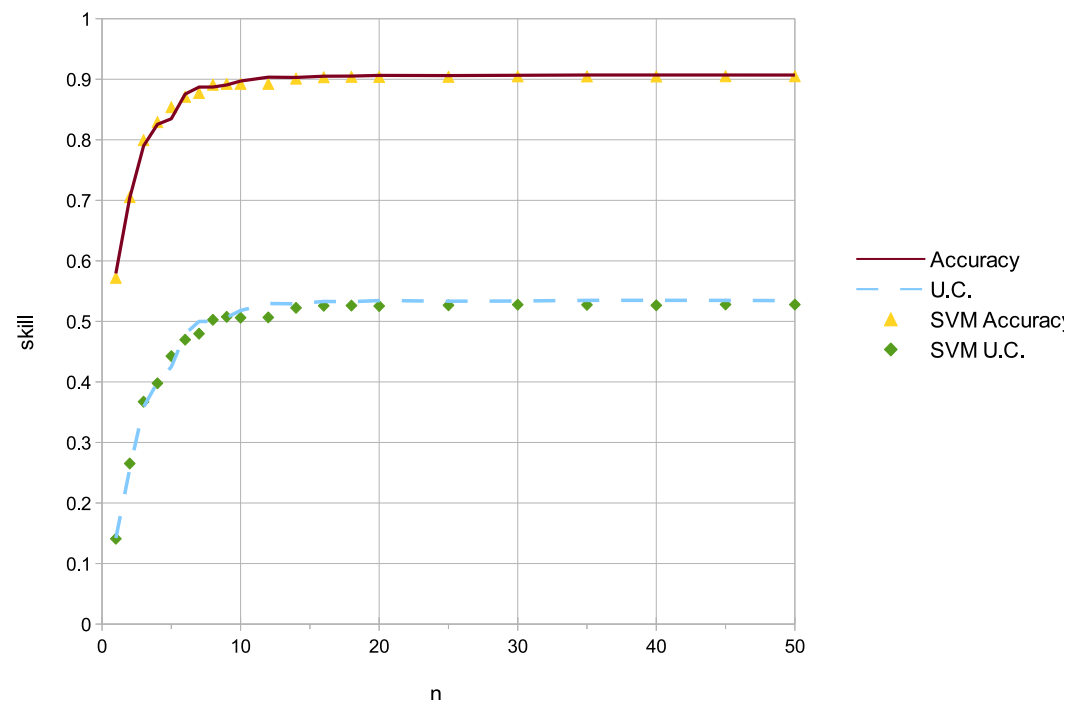

Figure 3: Classification accuracy and uncertainty coefficient for borderclassification starting with probabilities from the class definitions, and a support vector machine (SVM). Average of 20 trials. 


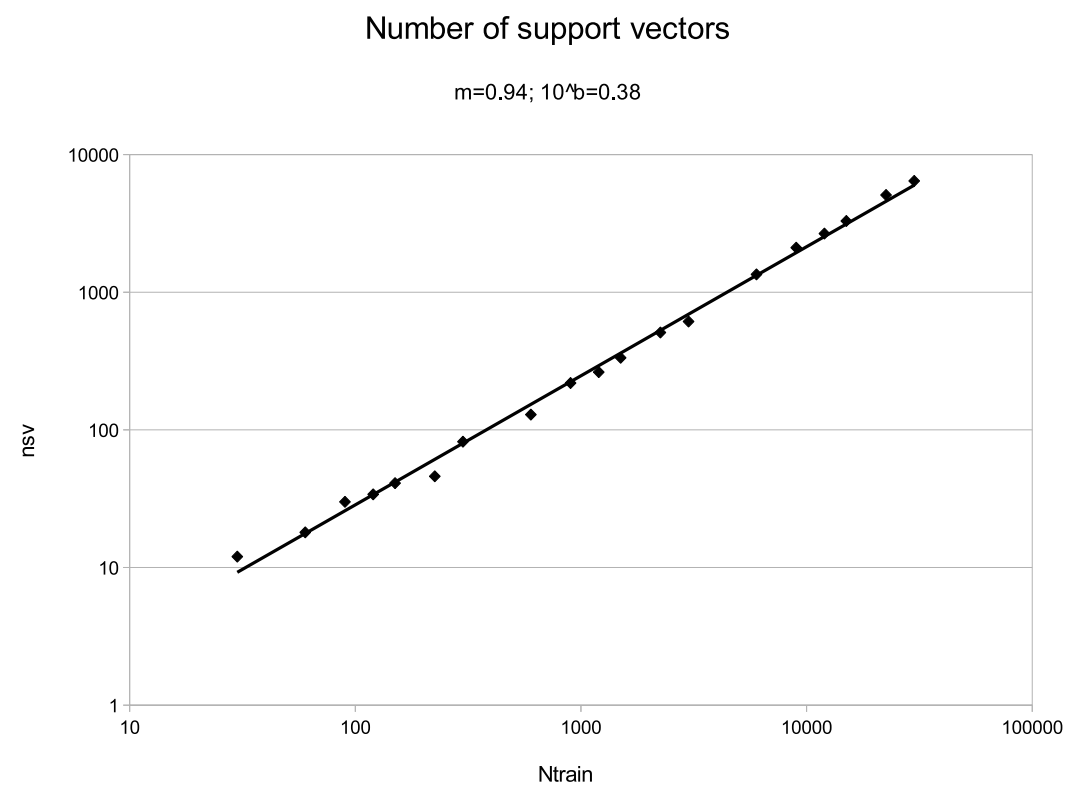

Figure 4: Number of support vectors against number of training vectors for pair of synthetic test classes. Fitted curve returns exponent of 0.94 and multiplication coefficient of 0.38 .

skill as a function of border samples, Figure 3 shows skill as a function of the number of border samples for a both an SVM-trained border classifier as well as a border classifier trained from the mathematical definition of the classes themselves. Note that both curves are roughly the same. So long as the complexity of the problem does not increase, adding new training samples does not increase the number of border samples required for maximum accuracy.

Figure 4 shows the number of support vectors versus the number of training samples. The fitted curve is approximately linear with an exponent of 0.94 and multiplication coefficient of 0.38 . In other words, for this problem there will be approximately $38 \%$ as many support vectors as there are training vectors.

Of course it's possible to speed up an SVM by sub-sampling the training data or the resulting support vectors. In such case, the sampling must be done carefully so as not to reduce the accuracy of the result. Figure 5 shows the effect on classification skill for the synthetic test classes when the number 
Skill versus number of training samples

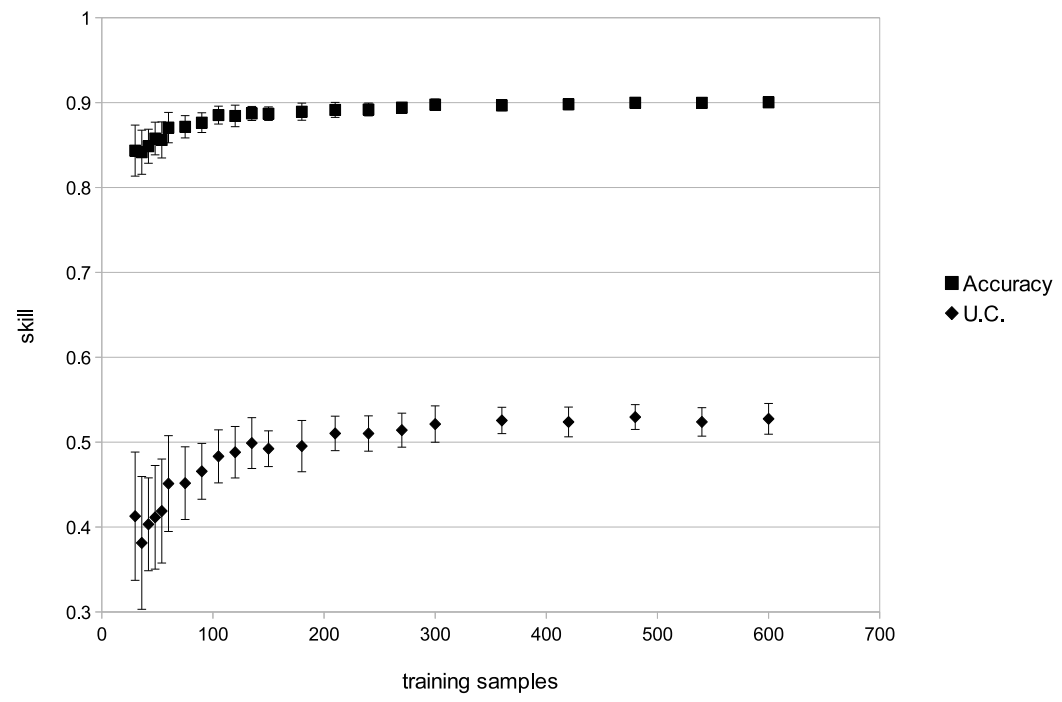

Figure 5: Classification accuracy and uncertainty coefficient for a support vector machine (SVM) trained with different numbers of samples. Error bars represent the standard deviation of 20 trials.

of training samples is reduced. Skill scores start to saturate at between 200 and 300 samples. By contrast, Figure 3 implies that you need only 20 border samples for good accuracy, so even with only 200 training samples you will still have improved efficiency by using the borders technique.

This suggests a simple scaling law. The number of training samples required for good accuracy, and hence the number of support vectors, should be proportional to the approximate volume occupied by the training data in the feature space: $n_{0} \propto V$ where $n_{0}$ is the minimum number of training vectors and $V$ is volume. Then the number of border vectors should be proportional to the volume taken to the root of the dimension of the feature space then raised to the power of one less: $n_{b 0} \propto V^{\frac{1}{D-1}}$. Putting it together, we can relate the two as follows: 1

\footnotetext{
${ }^{1}$ The correct form of the relation is: $n_{b 0} \propto n_{0}^{\frac{D-1}{D}}$. This footnote does not appear in the original article.
} 


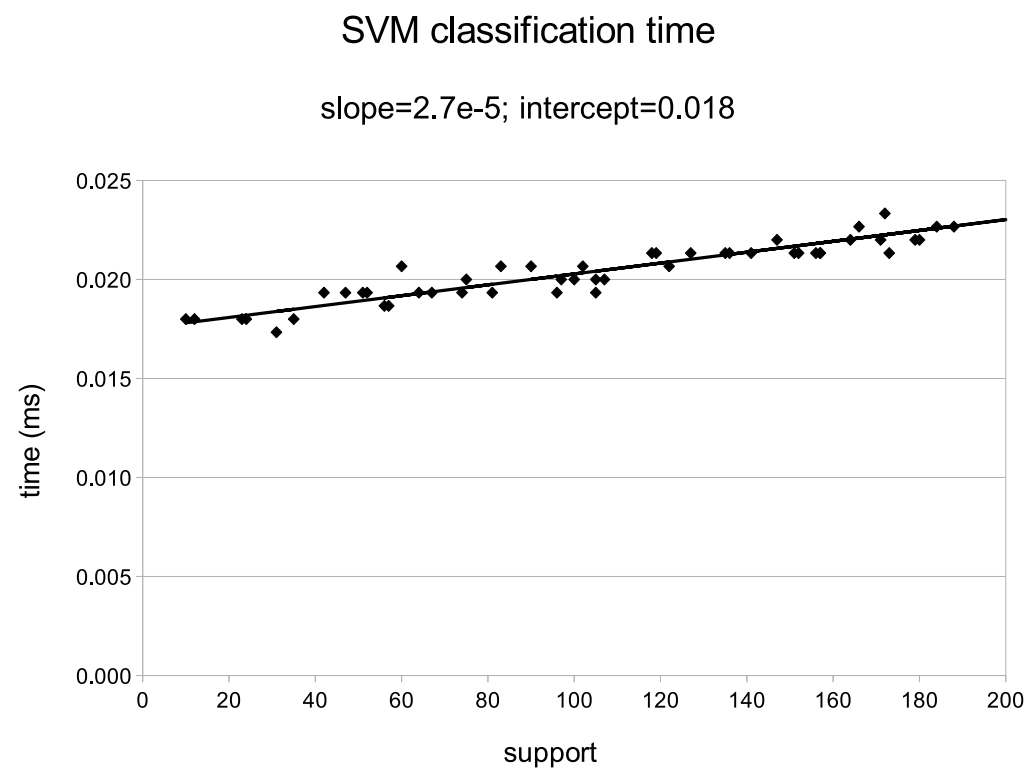

Figure 6: Classification time for a SVM for a single test point versus number of support vectors.

$$
n_{b 0} \propto n_{0}^{\frac{1}{D-1}}
$$

where $n_{b 0}$ is the minimum number of border vectors required for good accuracy.

In other words, provided the class borders are not fractal ( $\mathrm{Ott}, 1993$ ), mapping only the border between classes should always be faster than techniques that map the entirety of the class locations, although the advantage becomes less the larger the dimension of the feature space. This includes kernel density methods including SVM as well as similar methods such as learning vector quantization (LVQ) (Kohonen, 2000; Kohonen et al., 1995) that attempt to create an idealized representation of the classes through a set of "codebook" vectors.

To make this more concrete, Figure 6 plots the classification time versus the number of support vectors for a SVM while Figure 7 plots the classification time versus the number of border samples for a border classifier. 


\section{Borders classification time}

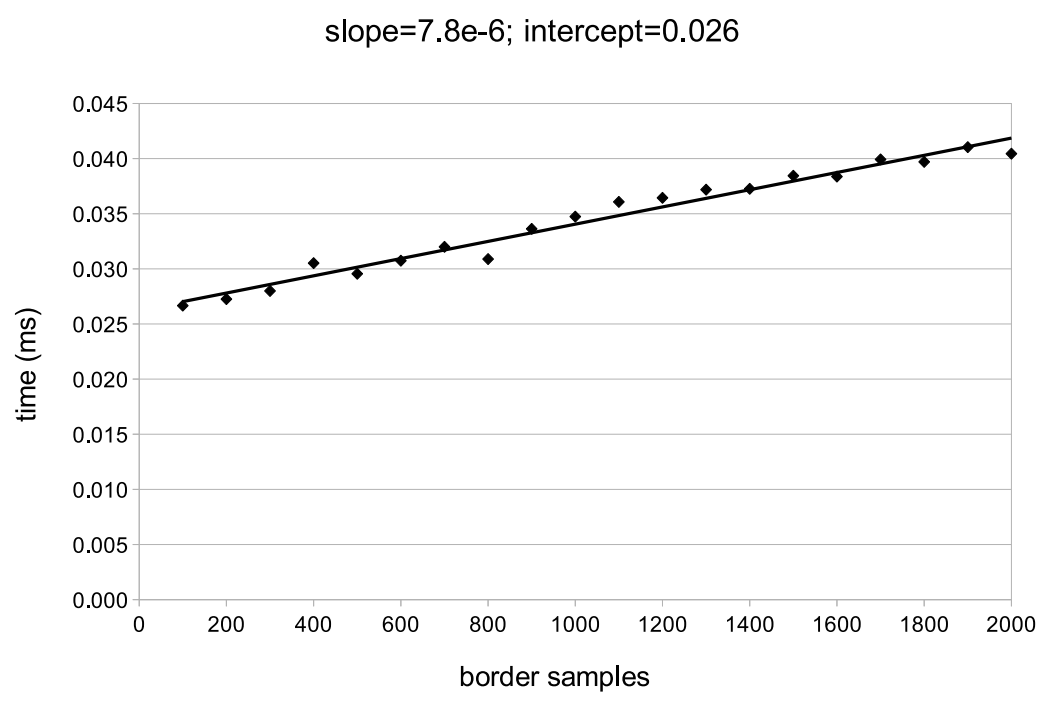

Figure 7: Classification time for a border classifier for a single test point versus number of border samples. 


\section{Border samples at break even}

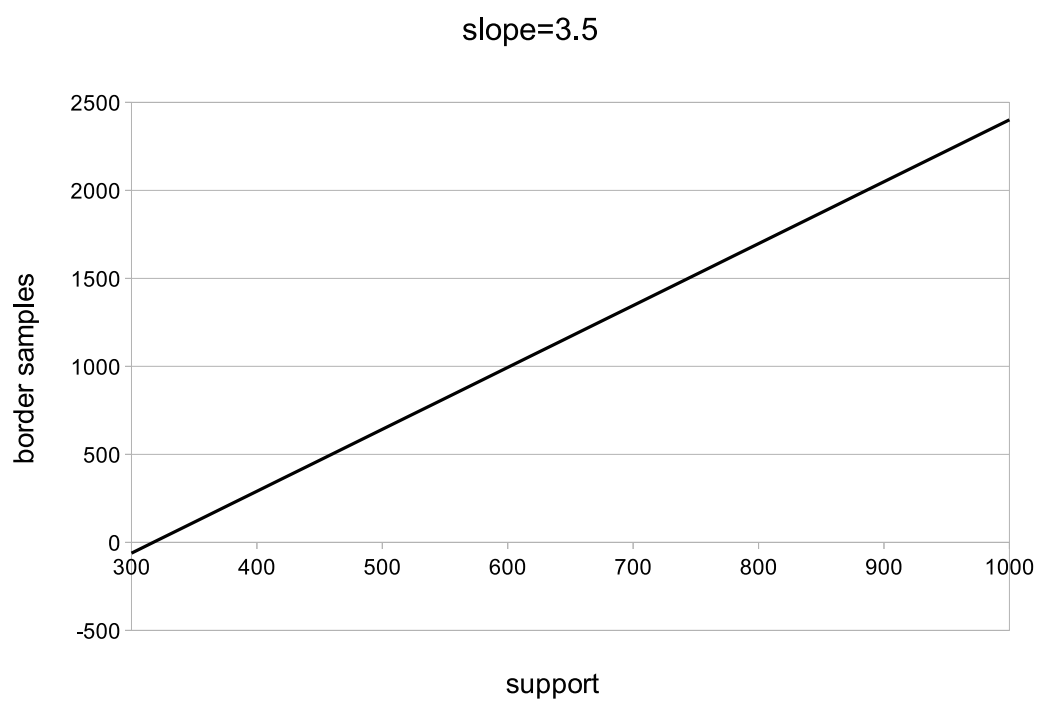

Figure 8: Number of border samples versus number of support vectors for equal classification times.

Classification times are for a single test point. Fitted straight lines are overlaid for each and the slope and intercept printed in the subtitle.

Figure 8 plots the number of border vectors versus the number of support vectors at the "break even" point: that is, the classification time is the same for each method. This graph was simply derived from the fitted coefficients of the previous two graphs. It is somewhat optimistic since LIBSVM has a larger overhead than the border classifiers. This overhead would be less significant for larger problems with the "rule of thumb" suggested by the slope that the number of border vectors should be less than three times the support for a reasonable gain in efficiency.

Unfortunately the graph is not general: while the borders method scales linearly with the number of classes, in LIBSVM there is some shared calculation for multi-class problems. That is, some of the support vectors are shared between classes moreover the number will be different for each problem. Model size comparisons between the two methods should ideally be between the total number of support vectors versus the total number of border vectors, not border (or support) vectors per class. Both methods 
will tend to scale linearly with the number of attributes, with a small component independent but a different amount for each method. Once we take into account the number of classes and number of attributes, the model for time complexity becomes quite complex so no attempt will be made here to fit it.

\section{Case studies}

Three classification models were tested on each of the 17 datasets described in Section 3.4 a basic linear classifier, a support vector machine (SVM) and a borders model derived from the previous SVM model (Accel. for "accelerated" SVM). SVM should be more accurate than linear classification for problems in which the classes are not linearly separable. Meanwhile, for large problems, the borders technique should produce significant time savings over SVM while having little effect on accuracy.

The parameters used for each method are summarized in Table 2, The parameter, $n_{b}$, in the borders technique was chosen for the best compromise between reduced accuracy and a speed improvement over SVM.

For SVM, a Gaussian kernel, also known as a radial basis function (RBF), is used:

$$
K(\vec{x}, \vec{y})=\exp \left(-\gamma|\vec{y}-\vec{x}|^{2}\right)
$$

where $\gamma$ is a tunable parameter. $C$ is a cost parameter added to reduce over-fitting: see Equations (44) and (5).

In order to get a confidence interval on the results, ten trials were performed for most of the datasets. In some cases, only a single trial was performed either because the operation took too long or because of a preexisting separation between test and training data which was taken "as is". Single trials are indicated through the absence of error bars which are calculated from the standard deviation. $f$ is the fraction of test data relative to the total number of samples.

The results are summarized in Tables 3 and 4 including training and test time for each method as well as skill scores. There are two skill scores, the first being simple accuracy or fraction of correct guesses while the second, called the uncertainty coefficient, is based on information entropy and is described in Section 2.4. The best values for each dataset are highlighted in bold. The SVM-borders method is not a stand-alone method thus its training time is never highlighted.

All but one of the classification problems show a significant speed increase with the application of the borders technique with letter being the 
Table 2: Summary of the parameters used in the numerical trials for each of the three methods: Linear, SVM (support vector machine) and ACC ("accelerated" SVM).

\begin{tabular}{|l|ll|l|ll|l|}
\hline Dataset & Stat. & & Linear & SVM & & Accel. \\
\hline & trials & $f$ & type $^{+}$ & $\gamma$ & $\mathrm{C}$ & $n_{b}$ \\
\hline \hline heart & 10 & 0.4 & 1 & 0.01 & 0.5 & 100 \\
shuttle & $10^{*}$ & 0.25 & 4 & 0.111 & 1 & 100 \\
sat & $10^{*}$ & 0.31 & 4 & 0.1 & 50 & 200 \\
segment & 10 & 0.4 & 4 & 0.1 & 100 & 50 \\
dna & $10^{*}$ & 0.372 & 2 & 0.0055 & 1 & 1000 \\
splice & $10^{*}$ & 0.685 & 1 & 0.00167 & 1 & 500 \\
codrna & $10^{*}$ & 0.82 & 4 & 0.125 & 1 & 500 \\
letter & $10^{*}$ & 0.4 & 4 & 0.065 & 1 & 75 \\
pendigits & $10^{*}$ & 0.318 & 4 & 0.01 & 50 & 200 \\
usps & $10^{*}$ & 0.216 & 4 & 0.004 & 1 & 50 \\
mnist & 1 & 0.143 & 1 & 0.0015 & 50 & 500 \\
ijcnn1 & $10^{*}$ & 0.647 & 0 & 0.045 & 1 & 500 \\
madelon & $10^{*}$ & 0.231 & 2 & 0.002 & 1 & 100 \\
seismic & $10^{*}$ & 0.2 & 1 & 0.02 & 1 & 200 \\
mushrooms & 10 & 0.4 & 1 & 0.0089 & 50 & 200 \\
phishing & 10 & 0.4 & 0 & 0.00147 & 1 & 500 \\
humidity & $10^{*}$ & 0.4 & 5 & 0.143 & 50 & 200 \\
\hline
\end{tabular}

* Some operations received only a single trial. See text.

+ Key:(Fan et al., 2008)

0. L2-regularized logistic regression, primal optimization

1. L2-regularized L2-loss SVM, dual optimization

2. L2-regularized L2-loss SVM, primal optimization

3. L2-regularized L1-loss SVM, dual optimization

4. SVM by Crammer and Singer (Crammer and Singer, 2002)

5. L1-regularized L2-loss SVM 
Table 3: Collation of results for numerical trials of the three different statistical classification methods over seventeen different datasets.

\begin{tabular}{|ll|lll|}
\hline dataset & quantity & Linear & SVM & Accel. \\
\hline \hline heart & train (s) & $\mathbf{0 . 0 2 3} \pm \mathbf{0 . 0 1 3}$ & $0.062 \pm 0.015$ & $0.060 \pm 0.009$ \\
& test (s) & $\mathbf{0 . 0 2 1} \pm \mathbf{0 . 0 1 1}$ & $0.030 \pm 0.009$ & $0.027 \pm 0.012$ \\
& acc & $0.827 \pm 0.024$ & $\mathbf{0 . 8 2 9} \pm \mathbf{0 . 0 2 2}$ & $0.829 \pm 0.022$ \\
& U.C. & $0.337 \pm 0.054$ & $\mathbf{0 . 3 4 1} \pm \mathbf{0 . 0 5 4}$ & $0.341 \pm 0.054$ \\
\hline shuttle & train (s) & $96 \pm 7$ & $\mathbf{8 8} \pm \mathbf{4}$ & $2.33 \pm 0.05$ \\
& test (s) & $\mathbf{0 . 2 2} \pm \mathbf{0 . 0 1}$ & $5.1 \pm 0.1$ & $2.60 \pm 0.05$ \\
& acc & 0.813 & $\mathbf{0 . 9 9 8}$ & $0.996 \pm 0.001$ \\
& U.C. & 0.607 & $\mathbf{0 . 9 8 1}$ & $0.979 \pm 0.005$ \\
\hline sat & train (s) & $\mathbf{0 . 9 9} \pm \mathbf{0 . 0 5}$ & $8.7 \pm 0.4$ & $8.1 \pm 0.2$ \\
& test (s) & $\mathbf{0 . 0 8 3} \pm \mathbf{0 . 0 1 7}$ & $1.01 \pm 0.07$ & $0.85 \pm 0.03$ \\
& acc & 0.827 & $\mathbf{0 . 9 1 4}$ & $0.889 \pm 0.004$ \\
& U.C. & 0.679 & $\mathbf{0 . 8 0 0}$ & $0.764 \pm 0.007$ \\
\hline segment & train (s) & $\mathbf{0 . 1 2} \pm \mathbf{0 . 0 3}$ & $0.78 \pm 0.05$ & $0.98 \pm 0.04$ \\
& test (s) & $\mathbf{0 . 0 3 4} \pm \mathbf{0 . 0 1 8}$ & $0.25 \pm 0.02$ & $0.235 \pm 0.015$ \\
& acc & $0.933 \pm 0.008$ & $\mathbf{0 . 9 6 2} \pm \mathbf{0 . 0 0 9}$ & $0.956 \pm 0.006$ \\
& U.C. & $0.881 \pm 0.014$ & $\mathbf{0 . 9 1 7} \pm \mathbf{0 . 0 1 7}$ & $0.909 \pm 0.013$ \\
\hline dna & train (s) & $\mathbf{0 . 5 2} \pm \mathbf{0 . 0 3}$ & $18.2 \pm 1.1$ & $21.3 \pm 0.4$ \\
& test (s) & $\mathbf{0 . 1 3} \pm \mathbf{0 . 0 2}$ & $1.8 \pm 0.2$ & $1.19 \pm 0.02$ \\
& acc & 0.900 & $\mathbf{0 . 9 5 0}$ & $0.737 \pm 0.004$ \\
& U.C. & 0.684 & $\mathbf{0 . 7 8 0}$ & $0.472 \pm 0.008$ \\
\hline splice & train (s) & $\mathbf{0 . 2 4} \pm \mathbf{0 . 0 4}$ & $1.9 \pm 0.2$ & $1.6 \pm 0.1$ \\
& test (s) & $\mathbf{0 . 1 0 1} \pm \mathbf{0 . 0 0 9}$ & $0.64 \pm 0.07$ & $0.15 \pm 0.02$ \\
& acc & 0.846 & $\mathbf{0 . 8 9 4}$ & $0.768 \pm 0.003$ \\
& U.C. & 0.385 & $\mathbf{0 . 5 1 4}$ & $0.321 \pm 0.008$ \\
\hline pendigits & train (s) & $\mathbf{0 . 5 0} \pm \mathbf{0 . 0 3}$ & $3.71 \pm 0.13$ & $1.85 \pm 0.06$ \\
& test (s) & $\mathbf{0 . 1} \pm \mathbf{0 . 0 1 2}$ & $1.24 \pm 0.04$ & $1.12 \pm 0.05$ \\
& acc & 0.908 & $\mathbf{0 . 9 7 8}$ & $0.975 \pm 0.001$ \\
& U.C. & 0.831 & $\mathbf{0 . 9 5 0}$ & $0.945 \pm 0.002$ \\
\hline codrna & train (s) & $\mathbf{3 . 1 7} \pm \mathbf{0 . 1 2}$ & 929 & $9.6 \pm 0.2$ \\
& test (s) & $2.88 \pm 0.07$ & 346 & $\mathbf{2 . 7 0} \pm \mathbf{0 . 0 6}$ \\
& acc & 0.930 & $\mathbf{0 . 9 6 4}$ & $0.961 \pm 0.0004$ \\
& U.C. & 0.676 & $\mathbf{0 . 7 5 9}$ & $0.744 \pm 0.002$ \\
\hline train (s) & $\mathbf{3 . 0 0} \pm \mathbf{0 . 0 8}$ & $44.8 \pm 0.8$ & $31.9 \pm 0.7$ \\
& test (s) & $\mathbf{0 . 2 2} \pm \mathbf{0 . 0 2}$ & $16.1 \pm 0.8$ & $19.5 \pm 1.1$ \\
& acc & 0.723 & $\mathbf{0 . 9 6 3}$ & $0.929 \pm 0.0022$ \\
& U.C. & 0.637 & & \\
& & $\mathbf{0 . 9 3 5}$ & $0.882 \pm 003$ \\
\hline
\end{tabular}


Table 4: Collation of results for numerical trials of the four different statistical classification methods over seventeen different datasets.

\begin{tabular}{|c|c|c|c|c|}
\hline dataset & quantity & Linear & SVM & Accel. \\
\hline \multirow{4}{*}{ usps } & train $(\mathrm{s})$ & $18.3 \pm 0.4$ & $104 \pm 14$ & $36.4 \pm 1.6$ \\
\hline & test (s) & $0.305 \pm 0.015$ & $7.0 \pm 1.3$ & $4.1 \pm 0.3$ \\
\hline & acc & 0.898 & 0.949 & $0.940 \pm 0.0024$ \\
\hline & U.C. & 0.783 & 0.880 & $0.866 \pm 0.004$ \\
\hline \multirow[t]{4}{*}{ mnist } & $\operatorname{train}(\mathrm{s})$ & 11035 & 6381 & $1906 \pm 676$ \\
\hline & test (s) & 3.64 & 343 & $308 \pm 24$ \\
\hline & acc & 0.915 & 0.972 & $0.960 \pm 0.0008$ \\
\hline & U.C. & 0.806 & 0.924 & $0.900 \pm 0.002$ \\
\hline \multirow[t]{4}{*}{ ijcnn1 } & train $(\mathrm{s})$ & $1.00 \pm 0.04$ & 560 & $7.52 \pm 0.18$ \\
\hline & test $(\mathrm{s})$ & $1.62 \pm 0.03$ & 109 & $2.07 \pm 0.04$ \\
\hline & acc & 0.632 & 0.988 & $0.980 \pm 0.001$ \\
\hline & U.C. & 0.199 & 0.824 & $0.757 \pm 0.008$ \\
\hline \multirow[t]{4}{*}{ madelon } & train $(\mathrm{s})$ & $1.04 \pm 0.052$ & $86 \pm 6$ & $17.7 \pm 0.4$ \\
\hline & test $(\mathrm{s})$ & $0.193 \pm 0.016$ & $5.11 \pm 0.14$ & $0.094 \pm 0.015$ \\
\hline & acc & 0.578 & 0.578 & $0.580 \pm 0.007$ \\
\hline & U.C. & 0.0178 & 0.0178 & $0.0243 \pm 0.004$ \\
\hline \multirow[t]{4}{*}{ seismic } & $\operatorname{train}(\mathrm{s})$ & $4.8 \pm 0.2$ & 55905 & $74.0 \pm 0.6$ \\
\hline & test $(\mathrm{s})$ & $0.70 \pm 0.03$ & 350 & $2.37 \pm 0.05$ \\
\hline & acc & 0.648 & 0.724 & $0.695 \pm 0.004$ \\
\hline & U.C. & 0.292 & 0.308 & $0.275 \pm 0.004$ \\
\hline \multirow[t]{4}{*}{ mushrooms } & $\operatorname{train}(\mathrm{s})$ & $0.454 \pm 0.016$ & $33.1 \pm 0.8$ & $2.81 \pm 0.07$ \\
\hline & test $(\mathrm{s})$ & $0.229 \pm 0.014$ & $3.5 \pm 0.1$ & $0.18 \pm 0.02$ \\
\hline & acc & $1 . \pm 0.0003$ & $1 . \pm 0.0003$ & $0.998 \pm 0.002$ \\
\hline & U.C. & $0.999 \pm 0.003$ & $0.996 \pm 0.003$ & $0.985 \pm 0.017$ \\
\hline \multirow[t]{4}{*}{ phishing } & $\operatorname{train}(\mathrm{s})$ & $0.52 \pm 0.03$ & $39.3 \pm 0.9$ & $4.34 \pm 0.09$ \\
\hline & test $(\mathrm{s})$ & $0.22 \pm 0.02$ & $4.1 \pm 0.1$ & $0.32 \pm 0.02$ \\
\hline & acc & $0.939 \pm 0.002$ & $0.958 \pm 0.003$ & $0.952 \pm 0.003$ \\
\hline & U.C. & $0.664 \pm 0.009$ & $0.747 \pm 0.012$ & $0.721 \pm 0.012$ \\
\hline \multirow[t]{4}{*}{ humidity } & train $(\mathrm{s})$ & $16.4 \pm 1.8$ & 2712 & $71.1 \pm 1.6$ \\
\hline & test $(\mathrm{s})$ & $0.55 \pm 0.03$ & 236 & $9.2 \pm 0.2$ \\
\hline & acc & 0.441 & 0.609 & $0.605 \pm 0.0009$ \\
\hline & U.C. & 0.292 & 0.479 & $0.474 \pm 0.0005$ \\
\hline
\end{tabular}


Table 5: Total number of support vectors versus total number of border samples.

\begin{tabular}{|l|lllllll|}
\hline & $D$ & $n_{c}$ & $n$ & $\begin{array}{l}\text { Total } \\
\text { support }\end{array}$ & $\begin{array}{l}\text { Total } \\
\text { borders }\end{array}$ & $\begin{array}{l}\text { Time }(\mathrm{s}) \\
\text { SVM }\end{array}$ & $\begin{array}{l}\text { Time (s) } \\
\text { accel. }\end{array}$ \\
\hline heart & 13 & 2 & 162 & $111 \pm 5$ & 100 & 0.030 & 0.027 \\
shuttle & 9 & 7 & 43500 & 1277 & 2100 & 5.1 & 2.60 \\
sat & 36 & 6 & 4435 & 1447 & 3000 & 1.01 & 0.85 \\
segment & 19 & 7 & 1386 & $340 \pm 11$ & 1050 & 0.25 & 0.24 \\
dna & 180 & 3 & 2000 & 1288 & 1000 & 1.8 & 1.19 \\
splice & 60 & 2 & 1000 & 678 & 500 & 0.64 & 0.15 \\
codrna & 8 & 2 & 59535 & 8978 & 500 & 346 & 2.70 \\
letter & 112 & 26 & 12000 & 5355 & 5850 & 16.1 & 19.5 \\
pendigits & 16 & 10 & 7494 & 602 & 2250 & 1.24 & 1.12 \\
usps & 256 & 10 & 7291 & 2081 & 2250 & 7.0 & 4.11 \\
mnist & 665 & 10 & 45000 & 12282 & 22500 & 343 & 308 \\
ijcnn1 & 22 & 2 & 49990 & 4888 & 500 & 109 & 2.07 \\
madelon & 500 & 2 & 2000 & 1959 & 100 & 5.11 & 0.094 \\
seismic & 50 & 2 & 78823 & 44385 & 200 & 350 & 2.37 \\
mushrooms & 112 & 2 & 4874 & $1054 \pm 14$ & 200 & 3.5 & 0.18 \\
phishing & 68 & 2 & 6633 & $1445 \pm 20$ & 500 & 4.1 & 0.32 \\
humidity & 7 & 8 & 51840 & 37796 & 5600 & 236 & 9.2 \\
\hline
\end{tabular}

Table 6: Results from SVM trials after sub-sampling to match the SVMborders uncertainty coefficient score is matched.

\begin{tabular}{|l|llllll|}
\hline dataset & samples & S.V. & train $(\mathrm{s})$ & test $(\mathrm{s})$ & accuracy & U.C. \\
\hline shuttle & 6522 & $518 \pm 8$ & $3.6 \pm 0.2$ & $3.60 \pm 0.07$ & $0.997 \pm 0.001$ & $0.972 \pm 0.008$ \\
sat & 1772 & $733 \pm 13$ & $2.24 \pm 0.08$ & $0.70 \pm 0.04$ & $0.893 \pm 0.007$ & $0.766 \pm 0.010$ \\
segment & $1037 \pm 1$ & $290 \pm 9$ & $0.53 \pm 0.03$ & $0.229 \pm 0.009$ & $0.954 \pm 0.007$ & $0.903 \pm 0.012$ \\
dna & 99 & $98.9 \pm 0.3$ & $0.31 \pm 0.03$ & $0.30 \pm 0.01$ & $0.835 \pm 0.012$ & $0.478 \pm 0.032$ \\
splice & 169 & $158 \pm 3$ & $0.20 \pm 0.02$ & $0.29 \pm 0.03$ & $0.826 \pm 0.012$ & $0.334 \pm 0.026$ \\
codrna & 14883 & $2781 \pm 51$ & $51 \pm 4$ & $114 \pm 3$ & $0.961 \pm 0.0006$ & $0.741 \pm 0.003$ \\
pendigits & 4864 & $515 \pm 12$ & $2.23 \pm 0.06$ & $1.21 \pm 0.05$ & $0.975 \pm 0.002$ & $0.944 \pm 0.003$ \\
usps & 3276 & $1255 \pm 21$ & $31 \pm 2$ & $4.6 \pm 0.3$ & $0.941 \pm 0.003$ & $0.865 \pm 0.006$ \\
mnist & 22498 & 7729 & 2746 & 253 & 0.965 & 0.909 \\
ijcnn1 & $12596 \pm 315$ & $1805 \pm 52$ & $40 \pm 3$ & $34 \pm 1$ & $0.983 \pm 0.001$ & $0.744 \pm 0.012$ \\
seismic & 3934 & $2499 \pm 99$ & $29 \pm 3$ & $16 \pm 4$ & $0.705 \pm 0.003$ & $0.275 \pm 0.005$ \\
mushrooms & 1218 & $398 \pm 17$ & $2.4 \pm 0.3$ & $0.94 \pm 0.07$ & $0.997 \pm 0.001$ & $0.976 \pm 0.012$ \\
phishing & $2652.2 \pm 0.4$ & $769 \pm 11$ & $7.2 \pm 0.8$ & $1.5 \pm 0.1$ & $0.952 \pm 0.003$ & $0.719 \pm 0.012$ \\
humidity & $20637 \pm 1$ & $15162 \pm 73$ & $331 \pm 9$ & $83 \pm 3$ & $0.603 \pm 0.002$ & $0.471 \pm 0.002$ \\
\hline
\end{tabular}


lone exception. Despite its simplicity, linear classification is shown to be competitive with a non-parametric method such as SVM for many of the problems and it is almost always faster both for training and for testing. (For the author, at least, this is one of the more surprising results.)

Table 5 is an attempt to get a handle on the relative time complexity of the two methods-SVM and SVM-borders-and lists all the relevant variables: number of features, number of classes, total number of training samples, total support for SVM, total number of border samples for the borders method compared with the resulting classification time for the two methods. The two most relevant variables here are the number of support vectors versus the number of border vectors. In order to get a successful speed increase, the former should be larger than the latter, but as is apparent from some problems such as shuttle, pendigits, and usps, even having more border samples can sometimes produce a significant, although modest, improvement.

All increases in speed, however, come at the cost of accuracy. The question is, is the speed increase worth the decrease in skill? To test this, we sub-sample the datasets and then re-apply the SVM training until the skill the two methods, SVM and SVM-borders, as measured by the uncertainty coefficient matches.

It might seem more expedient to directly sub-sample the support vectors themselves rather than the training data. This, however, was found not to work and generated a precipitous drop in accuracy. Since the sparse coefficient set, $\vec{w}$, is found through simultaneous optimization, the support vectors turn out to be interdependent.

Depending on how much the dataset is reduced, sub-sampling should be done with at least some care. On one hand, a more sophisticated subsampling technique might be considered a method on its own, comparable with the borders technique, but also likely requiring multiple training phases using the original technique thus making it significantly slower. On the other hand, at minimum we should consider the relative size of each class distribution. If there are roughly the same number of classes, then for small subsamples the relative numbers should be kept constant. The shuttle dataset, however, has very uneven class numbers so it was sub-sampled differently in order to ensure that the smallest classes retain some membership. Let $n_{i}$ be the number of samples of the $i$ th class. Then the sub-sampled numbers are given:

$$
n_{i}^{\prime}=\alpha\left(n_{i}\right) n_{i}
$$

The form of $\alpha$ used for the shuttle dataset was:

$$
\alpha(n)=C n^{-\zeta}
$$


where $C=n_{1}^{\zeta}, 0<\zeta<1$ is determined based on the desired total fraction and $n_{1}$ is the number of samples in the smallest class. To understand how this functional form was chosen, please see Appendix A.

The results of the sub-sampling exercise are shown in Table6, This gives us a clearer understanding of whether or not and when SVM acceration through borders sampling is effective.

There are at least two major sources of error for the borders technique. First, it provides only limited sampling of the discrimination border and this sampling is not strongly optimized. The sampling method, using pairs of training points of opposite class, will tend to favour regions of high density, however directly optimizing for classification skill would be the ideal solution. Second, the probability estimates extrapolate from only a single point. These two errors will tend to compound, especially after converting to multiple classes.

\section{Conclusions}

The primary goal of this work was to improve the classification efficiency of a SVM using a simple, piecewise linear classifier which we call the borders classifier. The outcome for each of the 17 datasets is summarized in Table 7. When trained from the SVM, the method succeeded for twelve of the datasets. Of these, two return better results using a linear classifier rather than borders sampling. Not a perfect score but certainly worthwhile to try for operational retrievals where time performance is critical, for instance classifying large amounts of satellite data in real time. This is especially so in light of the high performance ratios for some of the problems: the humidity dataset is sped up by over 20 times, for instance, with even higher factors for some of the binary datasets.

The same analysis was not repeated for the linear classifier since it is a constant time algorithm, so should always come out the winner. Even so, in three of the trials, codrna, madelon and mushrooms, borders classifications was actually faster than a linear classifier. This may have more to do with implementation than anything else, however. If raw speed is the main criterion, linear classifiers are the gold standard.

Linear classification has the potential to be both fast and accurate over a broad range of datasets if the features are first mapped to a higher dimensional space, similar to polynomial regression fitting. This is done implicitly in SVM (Müller et al., 2001), but closed-form feature maps that are also efficient to compute are difficult to derive for many kernels, including RBF. 
Table 7: Summary of results for all 17 datasets including a verdict on the success or failure of borders classification to speed up SVM.

\begin{tabular}{|l|ll|ll|l|}
\hline dataset & time $(\mathrm{s})$ & & U.C. & verdict \\
& SVM & accel. & SVM & Accel. & \\
\hline heart & $0.030 \pm 0.009$ & $0.027 \pm 0.012$ & $0.34 \pm 0.05$ & $0.34 \pm 0.05$ & succeeds \\
shuttle & $3.60 \pm 0.07$ & $2.60 \pm 0.05$ & $0.972 \pm 0.008$ & $0.979 \pm 0.005$ & succeeds \\
sat & $0.70 \pm 0.04$ & $0.85 \pm 0.03$ & $0.77 \pm 0.01$ & $0.76 \pm 0.007$ & fails \\
segment & $0.229 \pm 0.009$ & $0.235 \pm 0.015$ & $0.90 \pm 0.01$ & $0.91 \pm 0.01$ & fails \\
dna & $0.30 \pm 0.01$ & $1.19 \pm 0.02$ & $0.48 \pm 0.03$ & $0.472 \pm 0.008$ & fails* \\
splice & $0.29 \pm 0.03$ & $0.15 \pm 0.02$ & $0.33 \pm 0.03$ & $0.321 \pm 0.008$ & succeeds* \\
codrna & $114 \pm 3$ & $2.70 \pm 0.06$ & $0.741 \pm 0.003$ & $0.744 \pm 0.002$ & succeeds \\
letter & $16.1 \pm 0.8$ & $19.5 \pm 1.1$ & 0.935 & $0.882 \pm 0.003$ & fails \\
pendigits & $1.21 \pm 0.05$ & $1.12 \pm 0.05$ & $0.944 \pm 0.003$ & $0.945 \pm 0.002$ & succeeds \\
usps & $4.6 \pm 0.3$ & $4.1 \pm 0.3$ & $0.865 \pm 0.006$ & $0.866 \pm 0.004$ & succeeds \\
mnist & 253 & $308 \pm 24$ & 0.909 & $0.900 \pm 0.002$ & fails \\
ijcnn1 & $34 \pm 1$ & $2.07 \pm 0.04$ & $0.74 \pm 0.01$ & $0.757 \pm 0.008$ & succeeds \\
madelon & $5.1 \pm 0.1$ & $0.09 \pm 0.02$ & 0.0178 & $0.0243 \pm 0.004$ & succeeds \\
seismic & $16 \pm 4$ & $2.37 \pm 0.05$ & $0.275 \pm 0.005$ & $0.275 \pm 0.004$ & succeeds \\
mushrooms & $0.94 \pm 0.07$ & $0.18 \pm 0.02$ & $0.98 \pm 0.01$ & $0.99 \pm 0.02$ & succeeds* \\
phishing & $7.2 \pm 0.8$ & $0.32 \pm 0.02$ & $0.72 \pm 0.01$ & $0.72 \pm 0.01$ & succeeds \\
humidity & $83 \pm 3$ & $9.2 \pm 0.2$ & $0.471 \pm 0.002$ & $0.474 \pm 0.0005$ & succeds \\
\hline
\end{tabular}

* Linear classification generates more accurate results. 
Nonetheless, Vedaldi and Zisserman (2012) have succeeded in doing so for several kernels popular in computer vision applications. Sadly, comparative results were not available in time.

It's worthwhile to note where the borders classification algorithm is most likely to succeed and conversely where it might fail. One of the most successful trials was for the humidity dataset which produced one of the largest time improvements combined with relatively little loss of accuracy. This makes sense since the method was devised specifically for this problem and the humidity dataset epitomizes the characteristics for which the technique is most effective.

Since it assumes that the difference in conditional probabilities is a smooth and continuous function, the borders method tends to work poorly with integer or categorical data as well as problems with sharply defined, non-overlapping classes. Indeed, two of the problems where it took the biggest hit in accuracy, dna and splice, use binary and categorical data respectively.

Also, since there is no redundancy in calculations for multiple classes, whereas in SVM there is considerable redundancy, problems with a large number of classes should also be avoided. This can be mitigated by using a multi-class classification method requiring fewer binary classifiers such as one-versus-the-rest with $O\left(n_{c}\right)$ performance or a decision tree with $O\left(\log n_{c}\right)$ performance, rather than one-versus-one with its $O\left(n_{c}^{2}\right)$ time complexity.

The most important characteristic for success with the borders classification method is a large number of training samples used to train a SVM for maximum accuracy. This also implies a large number of support vectors, making the SVM slow. Choosing an appropriate number of border samples allows one to trade off accuracy for speed, with diminishing returns for larger numbers of border samples. The borders method, unlike SVM, also has a straightforward interpretation: the location of the samples represent a hyper-surface that divides the two classes and their gradients are the normals to this surface. In this regard it is somewhat similar to rule-based classifiers such as decision trees.

There are many directions for future work. An obvious refinement would be to distribute the border samples less randomly and cluster them where they are most needed. As it is, the method of choosing by selecting random pairs of opposite classes, will tend to distribute them in areas of high density. The current, random method was found to work well enough. Another potential improvement would be to position the border samples so as to directly minimize classification error. This need not be done all at once as in some of the methods mentioned in the Introduction, but rather point- 
by-point to keep the training relatively fast. A first guess could be found through a kernel method and then each pointed shifted along the normal. Piecewise linear statistical classification methods are simple, powerful and fast and we think they should receive more attention.

For certain types of datasets, particularly those with continuum features data, smooth probability functions (typically overlapping classes) and a large number of samples, the borders classification algorithm is an effective method of improving the classification time of kernel methods. Because it is not a stand-alone method, but requires probability estimates, it can acheive a fast training time since it is not solving a global optimization problem, yet still maintain reasonable accuracy. While it may not be the first choice for cracking "hard" problems, it is ideal for workaday problems, such as operational retrievals, for which speed is critical.

\section{A Sub-sampling}

Let $n_{i}$ be the number of samples of the $i$ th class such that:

$$
n_{i} \geq n_{i-1}
$$

Let $0 \leq \alpha(n) \leq 1$ be a function used to sub-sample each of the class distributions in turn:

$$
n_{i}^{\prime}=\alpha\left(n_{i}\right) n_{i}
$$

We wish to retain the rank ordering of the class sizes:

$$
\alpha\left(n_{i}\right) n_{i} \geq \alpha\left(n_{i-1}\right) n_{i-1}
$$

while ensuring that the smallest classes have some minimum representation:

$$
\alpha\left(n_{i}\right) \leq \alpha\left(n_{i-1}\right)
$$

Thus:

$$
\begin{aligned}
\frac{\mathrm{d}}{\mathrm{d} n}[n \alpha(n)] & =\alpha(n)+n \frac{\mathrm{d} \alpha}{\mathrm{n}} \geq 0 \\
\frac{\mathrm{d} \alpha}{\mathrm{d} n} & \geq-\frac{\alpha(n)}{n}
\end{aligned}
$$

The simplest means of ensuring that both (27) and (28) are fulfilled, is to multiply the right side of (28) with a constant, $0 \leq \zeta \leq 1$, and equate it with the left side:

$$
\frac{\mathrm{d} \alpha}{\mathrm{d} n}=-\frac{\zeta \alpha(n)}{n}
$$


Integrating:

$$
\alpha(n)=C n^{-\zeta}
$$

The parameter, $C$, is set such that $n_{1}^{\prime}=n_{1}$ :

$$
C=n_{1}^{\zeta}
$$

while $\zeta$ is set such that:

$$
\begin{aligned}
f \sum_{i} n_{i} & =\sum_{i} \alpha\left(n_{i}\right) n_{i} \\
& =n_{1}^{\zeta} \sum_{i} n_{i}^{1-\zeta}
\end{aligned}
$$

where $0<f<1$ is the desired fraction of training data. 


\section{References}

Alimoglu, F. (1996). Combining Multiple Classifiers for Pen-Based Handwritten Digit Recognition. Master's thesis, Bogazici University.

Bagirov, A. M. (1999). Derivative-free methods for unconstrained nonsmooth optimization and its numerical analysis. Invstigacao Operacional, 19:75-93.

Bagirov, A. M. (2005). Max-min separability. Optimization Methods and Software, 20(2-3):277-296.

Chang, C.-C. and Lin, C.-J. (2011). LIBSVM: A library for support vector machines. ACM Transactions on Intelligent Systems and Technology, $2(3): 27: 1-27: 27$.

Crammer, K. and Singer, Y. (2002). On the Learnability and Design of Output Codes for Multiclass Problems. Machine Learning, 47(2-3):201233.

Duarte, M. F. and Hu, Y. H. (2004). Vehicle classification in distributed sensor networds. Journal of Parallel Distributed Computing, 64:826-838.

Fan, R.-E., Chang, K.-W., Hsieh, C.-J., Wang, X.-R., and Lin, C.-J. (2008). LIBLINEAR: A Library for Large Linear Classification. Journal of Machine Learning Research, 9:1871-1874.

Feldkamp, L. and Puskorius, G. V. (1998). A signal processing framework based on dynamic neural networks with application to problems in adaptation, filtering, and classification. Proceedings of the IEEE, 86(11):22592277 .

Frey, P. and Slate, D. (1991). Letter recognition using holland-style adaptive classifiers. Machine Learning, 6(2):161-182.

Gai, K. and Zhang, C. (2010). Learning Discriminative Piecewise Linear Models with Boundary Points. In Proceedings of the Twenty-Fourth AAAI Conference on Artificial Intelligence, pages 444-450. Association for the Advancement of Artificial Intelligence.

Guyon, I., Gunn, S., Hur, A. B., and Dror, G. (2004). Results analysis of the NIPS 2003 feature selection challenge. In Proceedings of the 17th International Conference on Neural Information Processing Systems, pages 545-552, Vancouver. MIT Press. 
Herman, G. T. and Yeung, K. T. D. (1992). On piecewise-linear classification. IEEE Transactions on Pattern Analysis and Machine Intelligence, 14(7):782-786.

Hsu, C.-W. and Lin, C.-J. (2002). A comparison of methods for multiclass support vector machines. IEEE Transactions on Neural Networks, 13(2):415-425.

Huang, X., Mehrkanoon, S., and Suykens, J. A. K. (2013). Support vector machines with piecewise linear feature mapping. Neurocomputing, 117(6):118-127.

Hull, J. J. (1994). A database for handwritten text recognition research. IEEE Transactions on Pattern Analysis and Machine Intelligence, 16(5):550-554.

Iba, W., Wogulis, J., and Lngley, P. (1988). Trading of simplicity and coverage in incremental concept learning. In Proceedings of Fifth International Conference on Machine Learning, pages 73-79.

King, R. D., Feng, C., and Sutherland, A. (1995). Statlog: Comparision of Classification Problems on Large Real-World Problems. Applied Artificial Intelligence, 9(3):289-333.

Kohonen, T. (2000). Self-Organizing Maps. Springer-Verlag, 3rd edition.

Kohonen, T., Hynninen, J., Kangas, J., Laaksonen, J., and Torkkola, K. (1995). LVQ PAK: The Learning Vector Quantization Package, Version 3.1.

Kostin, A. (2006). A simple and fast multi-class piecewise linear pattern classifier. Pattern Recognition, 39:1949-1962.

LeCun, Y., Bottou, L., Bengio, Y., and Haffner, P. (1998). Gradientbased learning applied to document recognition. Proceedings of the IEEE, 86(11):2278-2324.

Lee, T. and Richards, J. A. (1984). Piecewise linear classification using seniority logic committee methods with application to remote sensing. Pattern Recognition, 17(4):453-464.

Lee, T. and Richards, J. A. (1985). A low cost classifier for multitemporal applications. International Journal of Remote Sensing, 6(8):1405-1417. 
Lichman, M. (2013). UCI machine learning repository.

Lin, H.-T., Lin, C.-J., and Weng, R. C. (2007). A note on Platt's probabilistic outputs for support vector machines. Machine Learning, 68(267):276.

Michie, D., Spiegelhalter, D. J., and Tayler, C. C., editors (1994). Machine Learning, Neural and Statistical Classification. Ellis Horwood Series in Artificial Intelligence. Prentice Hall, Upper Saddle River, NJ. Available online at: http://www.amsta.leeds.ac.uk/ charles/statlog/.

Mills, P. (2009). Isoline retrieval: An optimal method for validation of advected contours. Computers \& Geosciences, 35(11):2020-2031.

Mills, P. (2011). Efficient statistical classification of satellite measurements. International Journal of Remote Sensing, 32(21):6109-6132.

Mohommad, R., Fadi Abdeljaber Thabtah, F. A., and McCluskey, T. (2014). Predicting phishing websites based on self-structuring neural network. Neural Computing and Applications, 25(2):443-458.

Müller, K.-R., Mika, S., Rätsch, G., Tsuda, K., and Schölkopf, B. (2001). An introduction to kernel-based learning algorithms. IEEE Transactions on Neural Networks, 12(2):181-201.

Osborne, M. (1977). Seniority Logic: A Logic of a Committee Machine. IEEE Transactions on Computers, 26(12):1302-1306.

Ott, E. (1993). Chaos in Dynamical Systems. Cambridge University Press.

Pavlidis, N. G., Hofmeyr, D. P., and Tasoulis, S. K. (2016). Minimum Density Hyperplanes. Journal of Machine Learning Research, 17(156):133.

Platt, J. (1999). Probabilistic outputs for support vector machines and comparison to regularized likelihood methods. In Advances in Large Margin Classifiers. MIT Press.

Press, W. H., Teukolsky, S. A., Vetterling, W. T., and Flannery, B. P. (1992). Numerical Recipes in C. Cambridge University Press, 2nd edition.

Shannon, C. E. and Weaver, W. (1963). The Mathematical Theory of Communication. University of Illinois Press. 
Sklansky, J. and Michelotti, L. (1980). Locally trained piecewise linear classifiers. IEEE Transactions on Pattern Analysis and Machine Intelligence, 2(2):101-111.

Tenmoto, H., Kuda, M., and Shimbo, M. (1998). Piecewsise linear classifiers with an appropriate number of hyperplanes. Pattern Recognition, 31(11):1627-1634.

Terrell, D. G. and Scott, D. W. (1992). Variable kernel density estimation. Annals of Statistics, 20:1236-1265.

Uzilov, A. V., Keegan, J. M., and Mathews, D. H. (2006). Detection of non-coding rnas on the basis of predicted secondary structure formation free energy change. BMC Bioinformatics, 7:173.

Vedaldi, A. and Zisserman, A. (2012). Efficient Additive Kernel via Explicit Feature Maps. IEEE Transaction on Pattern Analysis and Machine Intelligence, 34(3):480-492.

Wang, J. and Saligrama, V. (2013). Locally-Linear Learning Machines (L3M). In Proceedings of Machine Learning Research, volume 29, pages $451-466$.

Webb, D. (2012). Efficient Piecewise Linear Classifiers and Applications. $\mathrm{PhD}$ thesis, University of Ballarat, Victoria, Australia.

Wu, T.-F., Lin, C.-J., and Weng, R. C. (2004). Probability Estimates for Multi-class Classification by Pairwise Coupling. Journal of Machine Learning Research, 5:975-1005. 